\title{
How to turn yourself into a virtual travel companion in someone else's car: drawing design approaches from the philosophy of mind
}

\author{
Laura Boffi $^{1}$ \\ ${ }^{1}$ IDAUP, University of Ferrara, \\ via Quartieri n.8, 44121 Ferrara, Italia \\ bfflra@unife.it \\ boffilaura@gmail.com
}

\begin{abstract}
Technological advancements in the development of autonomous vehicles have been focusing on the very fundamental issues such as functionality and safety. On the other hand, design researchers have started to explore possible everyday life interactions between people and automated vehicles, as well as probing the acceptance of such a disruptive technology by people and the emergence of trust from humans towards such cars. In this paper, I speculate on the possibility that autonomous driving might enable new human-to-human relationships. Departing from the "Co-Drive" concept, which envisions a new way of travelling and socializing inside the car by sharing a car trip with a remote virtual companion, I focus on the design challenges into the creation of one's own virtual avatar who inhabits the car as the remote passenger and I draw possible design approaches into the construction of a (virtual) self-representation from the philosophy of mind.
\end{abstract}

Keywords: Autonomous Cars · Interaction Design · Tele-Immersion · Telepresence $\cdot$ Virtual Embodiment $\cdot$ Virtual Reality $\cdot$ Philosophy of Mind

\section{Introduction}

The recent technological advancements in the development of (semi) autonomous vehicles (AVs) have been focusing on the very fundamental issues such as driving functionality and safety. On the other hand, design researchers, human factor specialists, psychologists among others have started to explore possible everyday life interactions between people and automated vehicles, as well as probing the acceptance of such a disruptive technology by people and the emergence of trust from humans towards such cars $[1,2,3]$. Design Research has currently been focusing on new communication paradigms between people and autonomous vehicles, turning both the inside and the outside of the automated cars into interfaces towards passengers, pedestrians, and bikers. The envisioned concepts mainly propose the use of graphical and 
light-based interfaces to convey messages about the automated driving behaviour from the outside of the car to people in the streets [4].

In addition to car-to-human communication in an $\mathrm{AV}$ scenario, what most interests me as an interaction design researcher is the possibility that autonomous driving might enable new social, human-to-human relationships. The envisioned Co-Drive concept, which will be discussed in the following sections, stands as a design speculation on this opportunity area for design. According to Co-Drive, semi-autonomous cars will be able to host remote passengers as virtual avatars beside the human driver, as well as be driven from home by the remote passenger. In this paper I will firstly define the Co-Drive concept as a telepresence experience $[5,6]$ and describe its conceptual setup [7], and secondly I will give a brief overview of the philosophy of mind concepts that are related to the creation of own self-representation in virtual reality. As highlighted by philosopher T. Metzinger [8], theoretical concepts like embodiment, phenomenal self model (PSM), phenomenal unit of identification (UI) can be applied to the field of Virtual Reality to explore the design of VR experiences in a given context. In the conclusion of this paper, from such philosophy of mind theories I will draw a people centred design approach that would allow me to co-create with participants their digital self-representations along the Co-Drive journey.

\section{The Co-Drive concept}

Co-Drive is a future design concept where autonomous vehicles technologies are used beyond their core driving functionality and safety towards enabling new social interactions. It has been captured as a video scenario, which is available for watching online [9]. As stated by Carroll and Rosson [ 10, 11], scenarios are stories envisioned early and continuingly in the design process about people and their activities, as both enabled and constrained by the context and technologies. Scenarios offer descriptions about situations of use before situations are created and technologies are developed and are an effective tool which emphasize people experiences over technological functional specification. Technological objects are embedded in the scenarios as uncomplete props, or narrative placeholders [12], which suggests people possible uses, open up for conversation and feedback, and allow for technology appropriation by potential users.

Being the Co-Drive concept at its early development stage, I represented it as a video scenario for several reasons. A scenario based design has allowed me to focus my attention on co-existing contexts of use (the car, the city, the home) which stage and constrain the social interaction between the driver and the remote passenger. Moreover, I could rehearse possible interactions between the two people without spending any particular effort in the design of technology interfaces. I only built paper prototypes as narrative props that showed how technology would be embedded in the re- 
spective users contexts and their daily behaviours, and left all the functional description in the backstage. As a reflection tool, the scenario elicited a series of questions around virtual self-representation which has pushed me beyond static answers and generic assumptions toward the need of designing research probes and interventions in the wild [13], which are the object of this paper.

\subsection{The Co-Drive scenario}

When cars will be autonomous, people will still enjoy the experience of driving them at scattered times and of sharing the journey with other people as a social activity. As a matter of a fact, autonomous cars will allow drivers to detach from the driving and make the car trip a truly social experience by hosting a remote passenger for the duration or part of the journey. People at home will have a Co-Drive console and they will be able to create their own virtual representation as remote passengers, choosing from a low to high fidelity one, up to a photo-realistic hologram (Fig.1). Whenever a person at home feels like joining a car trip as the remote passenger, he can put his console on the Co-Drive mode, thus triggering the avatar he previously created of himself to stand and wait at the Co-Drive stop for a driver to pick him up for a ride. The remote passenger avatar at the Co-Drive bus stop will be visible to other drivers passing by through a windshield projection layered onto the real world. When approaching the avatar at the bus stop, the driver can receive additional information about the remote person it belongs to, thus deciding to host it in the car and share the car trip with him. If so, the person at home will get notified through the TV screen connected to the CoDrive console and will wear a VR helmet to start the remote trip in the car of the host driver. Both the driver and the remote passenger will be able to talk lively to each other for the duration of the trip. The remote passenger could switch with the driver and start driving remotely from home by using the console steering wheel. The autonomous car will always be able to correct trajectory and take the lead of the driving if needed. For example, senior citizens, who are losing their ability to drive and happen to spend lots of their time at home, could become frequent co-drivers for the sake of restoring their social life. They could enjoy travel back to affective places of their memories and drive again remotely with the intelligent assistance of the autonomous car. On the other side, the remote presence of the senior, mediated by the immersive technology, would facilitate the initial intergenerational relationship between the driver and the passenger. 

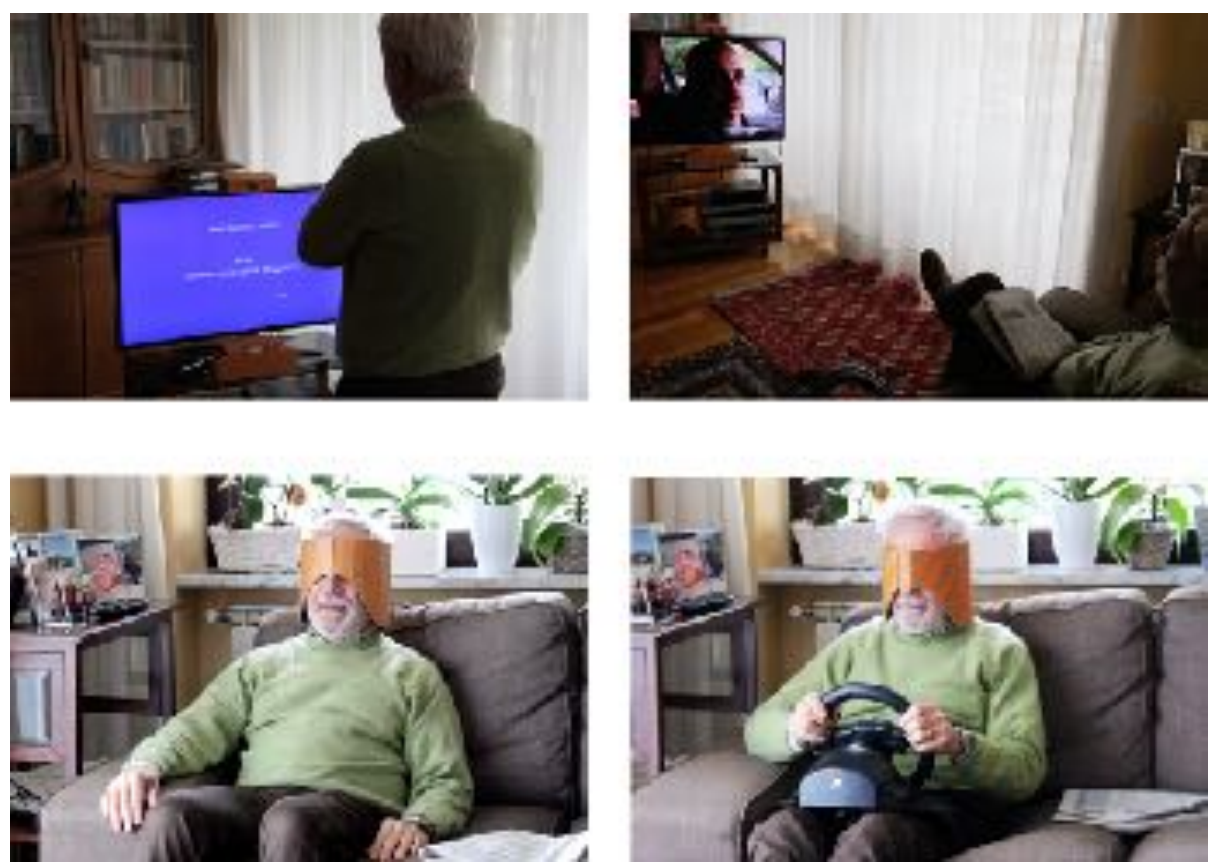

Fig. 1. Some scenes from the Co-Drive video scenario. Up to the left, the passenger recording his hologram; up to the right, the passenger being invited for a ride by a driver; down to the left, the passenger wearing the VR helmet and enjoying the journey; down to the right, the passenger taking the lead and driving from home.

\section{Immersive experience}

Co-Drive aims to immerse the remote passenger in a way that blurs the borders between his home and the physically distant environment of a car moving in the city. At the same time, it aims at delivering the driver the perception of having another person in the car to interact and share the journey with. Co-Drive aims to deliver a tele-immersion experience to the people involved, as defined by Ohl [7], so that they stay and perceive their own location; however at the same time, they are immersed in a shared car journey.

As I am interested in the experience delivered to people in this stage of my research, in this paper I will not focus on the technical details of the system, but on the conceptual characteristics of it. My final aim is to create the illusion to both the people, the driver and the host passenger, of "being there", as Marvin Minsky [14] used to mean telepresence when he first coined the term. Immersion has often been confused with presence. Slater [6] has tried to make the point and distinguished immersive as a quality of the technology, from presence, the experience felt by users [15]. According to Slater, immersion can be objectively assessed: the more a system is equipped with 
displays in all sensory modalities and tracking components which preserve sensorial fidelity in relation to their equivalent real world, the more it is "immersive". Virtual reality, for instance, is an immersive technology, used to create the subjective sense of presence. Presence instead should be conceived as a human reaction to an immersive system. We can design that technology to support presence as a human reaction, bearing in mind that the perception of "being present" is subjective from person to person. Different people might achieve presence with an immersive system that has low fidelity to reality (just visual wireframes or even through a simple telephone call for example), others would need high fidelity compared to the real world [5]. Virtual reality, for instance, is an immersive technology, used to create the subjective sense of presence in the user.

Thus, the broad question addressed in this paper could be expressed as following:

How might we enhance people subjective perception of presence within the Co-Drive scenario by the design of immersive systems?

In order to generate possible solutions to be tested with real people in an empiric way, a framework is needed to ground brainstorm and constrain ideas.

My suggestion is therefore to start with a conceptual description of the Co-Drive scenario as an immersive experience that would split it into its basic elements of communication and immersion, and thus to emancipate our thinking from a constrained technological perspective. The conceptual description of the Co-Drive scenario will help unlock opportunity areas for next design iterations, such as focusing on specific elements and interactions that need to be rethought or addressed in co-creation sessions with the users.

\subsection{Communication $\&$ immersive extent}

According to the description principle of tele-immersive concepts suggested by Ohl [7], Co-Drive presents 3 locations: 1. the passenger's home; 2 . the car; 3 . the city around the car. The city and the car are - for the driver and the remote passenger more or less the same location for the entire duration of the Co-Drive experience because the view of the world moves jointly with the car. Then, we can simplify saying that there are 2 locations, the car and the passenger's home, and 1 person at each location. Each location extends virtually to the other location and backwards, and each person can perceive the other. They can communicate over distance in a mutual way because they can talk to each other and they can see each other represented via scenes that are extracted from one location and sent to the other. Using Ohl notation, the communication can be described as $1<->1$, meaning that we have 1 person in each of the 2 physical locations; each location extends virtually to the other location and vice versa, making the system symmetric. 
For each communication channel, a different type of immersive extent can be defined. as a combination of scene extraction (portioning of the location to be sent virtually), warping (scaling or distortion of the virtual representation) and scene inclusion (positioning of the warped virtual representation in the local location). Representations of each location, what we refer to as scenes extraction, are sent to the other location, meaning from the passenger at home to the driver in the car and vice versa. In the CoDrive video scenario, I sketched some scenes while I managed to remain vague on other ones and to postpone any representational decisions to a later stage of the project, after fieldwork and co-creation with the users. The design decisions about how representing the self and the own location is infact the core of this paper.

For the driver in the car, I envisioned that a live video streaming of him and the car trip is taken from the passenger seat in the car and sent to the person at home. The person at home can remotely experience the car trip from the perspective of a passenger in the car.

About the passenger at home, I haven't sketched any visual representation to be sent to the driver in the car. I just envisioned that any representation, or scene, can be conveyed through windshield projections or holograms.

In both direction of communication, scene warping and inclusion haven't been left undefined intentionally and opened to speculation in this stage of the project. This openness is allowed by the design-driven approach of the project, which is not based on the adoption of any particular immersive technology.

Would people be willing to have a realistic representation of themselves at the CoDrive stop or would they opt for a simple wireframe of their silhouette? Which scale would they like to appear? Would people be willing to populate the car with objects from their living room? Those are just a few open questions for which I will need to run co-creation sessions with potential users, both drivers and passengers.

\section{Philosophy of mind and virtual self-representation}

In this section I will give a brief introduction of the philosophy of mind as a discipline of study. According to Lowe [16], the philosophy of mind is the study of minded beings - which he named as "subjects of experience" - which feel, perceive, think and perform intentional actions. Such beings include human persons, such as ourselves, who have bodies possessing various physical characteristics, such as height, weight and shape. More broadly, Lowe says that we can consider minded things all entities as long as they are minded, including animals and artificial beings like robots.

The philosophy of mind has been extensively focusing on the human mind-body relationship since Descartes and has tried to answer to the questions such as whether the mind is distinct from the body or some part of it, such as the brain, and whether the mind has properties, such as consciousness, which are unique to it. The mind-body 
problem, properly understood, is the problem of how minded beings - subjects of experience- are related to their physical bodies.

Philosopher Metzinger, among some others, has argued that conscious experience produced by biological nervous system is a virtual model of the world. According to this, the human brain produces a simulation of the physical world and events in it that the person -as a minded being or subject of experience- experiences consciously. The person doesn't acknowledge that what he perceives is "just" a simulation because only the content of the conscious representation is shown to him, and not the earlier processing stages of the construction process. Those stages are transparent and cannot be seen, thus the representation has phenomenal transparency [17]. According to Metzinger, virtual reality is nowadays the best technological metaphor for conscious experience, which is a property of the human mind. Virtual reality builds a construction of a possible world and possible selves and aims to deliver an experience of "presence" in the user, meaning a conscious experience in terms of the philosophy of mind. The concept of presence involves three dimensions:

1. Identification: being present as a self

2. Self-location in a temporal frame of reference: being present as a self now

3. Self-location in space: being present as a self now in this place

As it both happens in real life and in virtual reality, presence is experienced from a self-centered world and a first- person perspective of the surrounded world. Thus, everybody in real and virtual reality has his own private experience [18], which is particularly challenging to describe to other persons or to guess in other persons other than ourselves. This is what philosophers have been calling "the other-minds problem": the problem of gaining knowledge about another entity's mental state [8]. If as a private experience virtual reality doesn't shed any light on "the other-minds" problem, it is interesting how social VR [19] could potentially provide us with new means of acquiring concepts that we apply to other people other than ourselves and understanding how other people represent us in their mind.

Co-Drive stages a particular kind of social VR involving two persons at a time who share a car trip. It employs a certain degree of virtual representation for both the involved persons. As described in section 3, each location extends virtually to the other location and backward, and each person can perceive the other.

An interesting social VR experiment enabled by the Co-Drive concept could address the intergenerational relationship between the driver and the remote passenger and I will introduce it in the final section of this paper. I have already suggested that CoDrive could enable intergenerational relationships since elderly people could become frequent remote passengers for the sake of meeting new persons, travelling and shar- 
ing a trip, though virtual. How would a younger driver envision the virtual representation of their host older passenger? Would ageing stereotypes stick in the virtual world? How would senior passengers like to represent themselves to a younger person? Would they choose a realistic representation or would they create a custom avatar? Or would the two persons start a dynamic negotiation among themselves? Carrasco and others [20] have been already studying how elderly people are representing themselves in social virtual environments. I could contribute to the discourse focusing the attention on adults self-representations in the social frame of intergenerational relationships.

Another interesting issue to consider is how people can identify in their digital representation (their avatar) as their UI, unit of identification, instead of their own body. UI is defined as the phenomenal property with which a given subject self-identifies at a given time [8 Metz 2013]. At the moment, philosophers of mind consider that human beings identification with avatars is not fully achievable because our self-model is locked in our body and in our agency in controlling its actions. Our UI results in our own physical body. Anyway, interesting experiments could be created by manipulating the identity of a single body part [The sense of Embodiment, Slater] and synchronising portions of our body with our potential avatar. In this way we could investigate if partial artificial UIs could be achieved [8]. The famous rubber hand illusion is the classical experiment addressing the manipulation of a body part [21] and the creation of a form of identification.

Metzinger also defined minimal UI (the simplest possible unit of identification) as the experienced origin of the visuospatial perspective, regardless the body. A concrete example would be to map the person's gaze to the avatar, so to allow the person to control the avatar's visual attention. This would create a deep form of identification with the avatar because controlling one's own attention creates a sense of self on a mental level.

In the previous paragraph, I addressed the possibility of artificially creating a partial UI and I mentioned the rubber hand illusion. However, it doesn't provide a complete view into the embodiment, which philosophy of mind refers to as the subjective experience of owning and using a body [22]. According to Metzinger, as human beings we experience a model of the world and we inhabit a model of ourselves as well. As we live inside a model of our external world, whose rendering process we are not conscious of because it is not visible to us (the process is said to be "transparent"), at the same time we live inside models of our own bodies, minds and selves, the PSM- phenomenal self model [8]. Until now, our PSM has been able to inhabit exclusively our physical body and to be represented only through that, but the development of virtual reality is giving the possibility of owning another body, which is called virtual embodiment [6]. 
To allow complete sense of embodiment [21], the artificial body should deliver to the person the same properties of the biological body, such as:

$\begin{array}{ll}\text { 1. } & \text { Sense of self location } \\ \text { 2. } & \text { Sense of agency } \\ \text { 3. } & \text { Sense of body ownership }\end{array}$

One feels self located in the volume of her/his own body. It shouldn't be confused with the sense of "being there" (presence), which means being in a location without any reference to the inhabited body. First-person perspective is fundamental to enhance the sense of self-location inside a virtual body. Also vestibular and tactile information can support the sense of self location.

Synchronous movement of the real and virtual body enhances the sense of agency. Real body movements are mapped through to the real body and it is important that the visuomotor correlations are in real or near real time.

A person feels to own a body from a combination of influences: seen sensory simulations on the artificial body are felt synchronously on the human body and the artificial body resembles a human body. The creation of a personal avatar can enhance the sense of body ownership.

An interesting use of embodiment can be seen in the Freud experiment [23], where Slater and his team staged a VR experience in which a person was immersed in a psychotherapy session with psychoanalyst Freud. The person was firstly asked to speak out about a personal problem, then, after a moment of break, the person himself was embodied in Freud and was able to look at himself as a patient, listen to the personal problem he stayed some minutes earlier, and finally counselling himself as Freud. The person reported the experience to be enlightening because as Freud he was able to conceive suggestions that he would have never thought for himself. This happens because when people see a life-sized virtual body (VB) from first person perspective in virtual reality they are likely to have the perceptual illusion that it is their body. Additionally such virtual embodiment can lead to changes in perception, implicit attitudes and behaviour based on attributes of the VB [24].

\section{Design approaches for the Co-Drive scenario}

In section 3, I narrowed down the research question addressed in this paper:

How might we enhance people's subjective perception of presence within the CoDrive scenario by the design of immersive systems? 
Then, I described Co-Drive in terms of its conceptual parts (communication and immersion) and I introduced basic concepts from the philosophy of mind. In the next paragraphs I am going to unfold two opportunity areas [25] for the design of CoDrive which are based on philosophy of mind concepts, such as the "other-minds problem" and the minimal identification unit.

The opportunity areas will take into account that there could be two different, asymmetric self- representations of the driver and the host passenger, meaning that the way the passenger would represent himself to the driver would not correspond with the way he would see himself represented in the car from the VR goggles.

\subsection{Embodiment as a tool to demystify ageing stereotypes and to unfold the "other-minds problem"}

According to the Freud experiment, described in section 4, a VR experiment could be designed as a research activity to be carried in Co-Drive with the aim of probing digital representations of older people and challenge the perception of younger people about those representations.

An embodiment experiment could be staged as follows, involving the participation of a younger person as the driver. The younger person will be briefed about the Co-Drive concept first and then he will wear a VR set. He will see himself sharing a virtual CoDrive trip in a car with a senior passenger. Both of them will be represented with their digital avatars. The younger person will be prompted to start the conversation with the older person with the aim of inquiring what pushed the senior to participate in the CoDrive experience and become a remote passenger in the car. A break will happen now and when the experience will resume again, the younger person will be embodied in the remote older passenger. As the remote older passenger, the person will listen to the beginning of the conversation that he introduced some minutes earlier while embodied as the driver, and will answer to the questions as well.

With this experiment, I could draw insights on the aesthetics of ageing from a younger perspective, as well as I could capture genuine and unbiased motivations on the reasons to join a Co-Drive experience from the perspective of a younger person embodied in the older one. As a next step, the experiment could be revealed and played back to real older participants, capturing their subjective reactions on the motivations spoken by their avatar.

\subsection{Two ways -minimal UI to gain reciprocal eye contact}

If a body-resembling digital appearance is required to create the sense of embodiment, much less is needed to create a minimal identification. As described in section 3, a person can already feel identified with his avatar if he can experience the origin of the visuospatial perspective as if coming from his own eyes. In the Co-Drive concept, in 
order for the remote host passenger to reach the minimal identification with his representation, he would need to be able to turn his view inside the car as he turns his real face, and even to look for eye contact with the driver.

The minimum requirement for this to happen would consist of having the passenger gaze represented in the car and a camera placed in correspondence of the gaze which would broadcast to the remote passenger through VR goggles.

As an investigation, I could design a cultural probe [26] for participants asking them to take a picture of the eyes they would like to be represented with in the car, ranging from selfies to whatever their imagination can provide. Then I could run co-creation sessions with them envisioning how and where to embed those eyes in the car environment, as either a digital or a physical representation. One idea for example could be to have the eyes displayed from a rear view mirror, as a digital representation, or even having them embedded in a physical Lego-like robotic head hanging from the car ceiling on the passenger seat, as a physical representation.

\section{Conclusion}

In this paper I described the speculative design scenario Co-Drive, which focuses on a future social relationship enabled by autonomous cars technologies. I introduced some concepts related to the philosophy of mind and I tried to draw opportunity areas for the design of the Co-Drive experience from such concepts. Two initial opportunity areas are illustrated at the end of the paper, as well as two research activities which sparked out of those opportunities. The research activities would be carried out during the next phase of the project in order to explore and iterate the Co-Drive concept.

\section{References}

1. Strömberg, H., Pettersson, I., Andersson, J., Rydström, A., Dey, D., Klingegård, M., \& Forlizzi, J. (2018). Designing for social experiences with and within autonomous vehicles-exploring methodological directions. Design Science, 4.

2. Ward, C., Raue, M., Lee, C., D'Ambrosio, L. and Coughlin, J.F., (2017). Acceptance of automated driving across generations: The role of risk and benefit perception, knowledge, and trust. In International Conference on Human-Computer Interaction (pp. 254-266). Springer, Cham.

3. Frison, A.K., Aigner, L., Wintersberger, P. and Riener, A. (2018). Who is Generation A?: Investigating the Experience of Automated Driving for Different Age Groups. In Proceedings of the 10th International Conference on Automotive User Interfaces and Interactive Vehicular Applications (pp. 94-104). ACM.

4. Riegler, A., Wintersberger, P., Riener, A. and Holzmann, C. (2018). Investigating User Preferences for Windshield Displays in Automated Vehicles. In Proceedings of the 7th ACM International Symposium on Pervasive Displays (p. 8). ACM.

5. Steuer, J. (1992): Defining virtual reality: Dimensions determining telepresence. Journal of communication, 42(4), pp.73-93. 
6. Slater, M. (2003): A note on presence terminology. Presence connect. 2003 Jan;3(3):1-5.

7. Ohl, S. (2018): Tele-Immersion Concepts. IEEE transactions on visualization and computer graphics. Oct 1;24(10): 2827-42.

8. Metzinger, T.K. (2018): Why Is Virtual Reality Interesting for Philosophers?. Frontiers in Robotics and AI. 5:101.

9. Vimeo. (2019): Co-Drive. [Online Video]. 11 March 2018. Available from: https://vimeo.com/259302417. [Accessed: 11 February 2019].

10. Carroll, J.M. (2000): Making use: scenario-based design of human-computer interactions. MIT press.

11. Rosson, M.B. and Carroll, J.M. (2009). Scenario based design. Human-computer interaction. boca raton, FL, pp.145-162.

12. Halse, J., Brandt, E., Clark, B. and Binder, T. (2010). Rehearsing the future. The Danish Design School Press.

13. Smith, R.C., Vangkilde, K.T., Kjærsgaard, M.G., Otto, T., Halse, J. and Binder, T. eds. (2016): Design anthropological futures. Bloomsbury Publishing.

14. Minsky, M., 1980. Telepresence.

15. McCarthy, J. and Wright, P. (2004). Technology as experience. interactions, 11(5), pp. 42-43.

16. Lowe, E.J. (2000): An introduction to the philosophy of mind. Cambridge University Press.

17. Metzinger, T. (2003): Phenomenal transparency and cognitive self-reference. Phenomenology and the Cognitive Sciences, 2(4), pp.353-393.

18. Das, B. (2007): A Framework for Conscious Information Processing. arXiv preprint q-bio/ 0702033.

19. Schroeder, R. (2002): Social interaction in virtual environments: Key issues, common themes, and a framework for research. In The social life of avatars (pp. 1-18). Springer, London.

20. Carrasco, R., Baker, S., Waycott, J. and Vetere, F. (2017): Negotiating stereotypes of older adults through avatars. In Proceedings of the 29th Australian Conference on Computer-Human Interaction (pp. 218-227). ACM.

21. Kilteni, K., Groten, R. and Slater, M. (2012): The sense of embodiment in virtual reality. Presence: Teleoperators and Virtual Environments, 21(4), pp.373-387.

22. Blanke, O., and Metzinger, T. (2009): Full-body illusions and minimal phenomenal selfhood. Trends in Cognitive Sciences, 13(1), 7-13.

23. Freud-me. (2015): Freud-me. [ONLINE] Available at: http://freud-me.com/. [Accessed 11 February 2019].

24. Osimo, S. A., Pizarro, R., Spanlang, B., \& Slater, M. (2015): Conversations between self and self as Sigmund Freud-A virtual body ownership paradigm for self counselling. Scientific reports, 5, 13899.

25. Gaver, B., Dunne, T. and Pacenti, E. (1999): Design: cultural probes. interactions, 6(1), pp. 21-29. 